\title{
Factorization and Gauge Invariance of Twist-3 Cross Section for Single Spin Asymmetry
}

\author{
Yuji Koike $^{1}$ and Kazuhiro Tanaka ${ }^{2}$ \\ 1- Department of Physics, Niigata University, Ikarashi, Niigata 950-2181, Japan \\ 2- Department of Physics, Juntendo University, Inba-gun, Chiba 270-1695, Japan
}

\begin{abstract}
We prove factorization and gauge invariance of the twist- 3 single-spin-dependent cross section in the leading order perturbative QCD, which was missing in the previous literature. We emphasize that the consistency relation from the Ward identities for color gauge invariance is crucial to guarantee the cancelation among the various gaugenoninvariant contributions in the cross section. This relation also proves the absence of the "derivative" terms in the cross section corresponding to the hard-pole and softfermion-pole contributions. Applying the formalism to SIDIS, $e p^{\uparrow} \rightarrow e \pi X$, we have derived the complete cross section associated with the twist-3 distribution for the transversely polarized nucleon.
\end{abstract}

In the framework of the collinear factorization for hard inclusive processes, single spin asymmetry (SSA) in semi-inclusive reactions is a twist-3 observable and can be described by using certain quark-gluon correlation functions on the lightcone. In our recent paper [2], we have establised the twist-3 formalism for SSA, providing a proof for factorization property and gauge invariance of the single spin-dependent cross section in the leading order perturbative QCD, which was missing in the previous literature [3]-[10]. We also applied the method to derive the formula for SSA in semi-inclusive deep inelastic scattering (SIDIS), $e p^{\uparrow} \rightarrow e \pi X$. Here we shall state what had to be clarified for the twist-3 calculation in the previous literature, refering the readers to [2] for the details.

There are two independent twist-3 distribution functions for the transversely polarized nuleon. They can be defined in terms of the gluon's field strength $F^{\alpha \beta}$ as

$$
\begin{aligned}
& M_{F}^{\alpha}\left(x_{1}, x_{2}\right)_{i j} \\
& \quad=\int \frac{d \lambda}{2 \pi} \int \frac{d \mu}{2 \pi} e^{i \lambda x_{1}} e^{i \mu\left(x_{2}-x_{1}\right)}\left\langle p S_{\perp}\left|\bar{\psi}_{j}(0)[0, \mu n] g F^{\alpha \beta}(\mu n) n_{\beta}[\mu n, \lambda n] \psi_{i}(\lambda n)\right| p S_{\perp}\right\rangle \\
& \quad=\frac{M_{N}}{4}(\not p)_{i j} \epsilon^{\alpha p n S_{\perp}} G_{F}\left(x_{1}, x_{2}\right)+i \frac{M_{N}}{4}\left(\gamma_{5} \not p\right)_{i j} S_{\perp}^{\alpha} \widetilde{G}_{F}\left(x_{1}, x_{2}\right)+\cdots,
\end{aligned}
$$

where $\psi$ is the quark field, $p$ and $S_{\perp}$ are, respectively, momentum and spin vectors of the nucleon. $p$ can be regarded as light-like $\left(p^{2}=0\right)$ in the twist-3 accuracy, $n^{\mu}$ is the light-like vector $\left(n^{2}=0\right)$ with $p \cdot n=1$, and the spin vector satisfies $S_{\perp}^{2}=-1, S_{\perp} \cdot p=S_{\perp} \cdot n=0$. $[\mu n, \lambda n]$ is the gauge-link operator. The twist-3 distributions can also be defined in terms of the transverse component of the covariant dervative $D_{\perp}^{\alpha}=\partial_{\perp}^{\alpha}-i g A_{\perp}^{\alpha}$ as

$$
\begin{gathered}
\int \frac{d \lambda}{2 \pi} \int \frac{d \mu}{2 \pi} e^{i \lambda x_{1}} e^{i \mu\left(x_{2}-x_{1}\right)}\left\langle p S_{\perp}\left|\bar{\psi}_{j}(0)[0, \mu n] D_{\perp}^{\alpha}(\mu n)[\mu n, \lambda n] \psi_{i}(\lambda n)\right| p S_{\perp}\right\rangle \\
=\frac{M_{N}}{4}(\not p)_{i j} \epsilon^{\alpha p n S_{\perp}} G_{D}\left(x_{1}, x_{2}\right)+i \frac{M_{N}}{4}\left(\gamma_{5} \not p\right)_{i j} S_{\perp}^{\alpha} \widetilde{G}_{D}\left(x_{1}, x_{2}\right)+\cdots
\end{gathered}
$$

By introducing the nucleon mass $M_{N}$, the four functions $G_{F}, \widetilde{G}_{F}, G_{D}$ and $\widetilde{G}_{D}$ are defined as dimensionless. The " $F$-type" functions $\left\{G_{F}, \widetilde{G}_{F}\right\}$ and the "D-type" functions $\left\{G_{D}, \widetilde{G}_{D}\right\}$ 

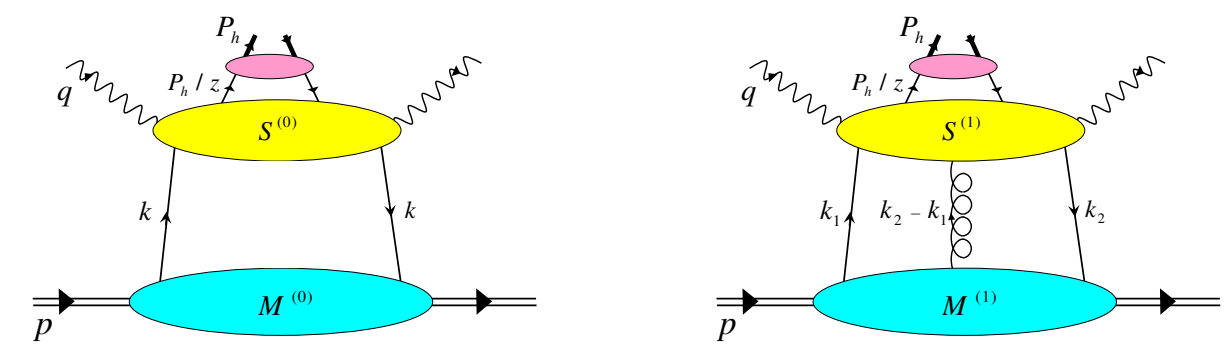

Figure 1: Generic diagrams for the hadronic tensor of $e p^{\uparrow} \rightarrow e \pi X$ which contribute in the twist-3 accuracy.

are not independent. They are related as [7]

$$
\begin{aligned}
G_{D}\left(x_{1}, x_{2}\right) & =P \frac{1}{x_{1}-x_{2}} G_{F}\left(x_{1}, x_{2}\right), \\
\widetilde{G}_{D}\left(x_{1}, x_{2}\right) & =\delta\left(x_{1}-x_{2}\right) \widetilde{g}\left(x_{1}\right)+P \frac{1}{x_{1}-x_{2}} \widetilde{G}_{F}\left(x_{1}, x_{2}\right),
\end{aligned}
$$

where $\widetilde{g}(x)$ is a function expressed in terms of $\left\{G_{F}, \widetilde{G}_{F}\right\}$ and the twist-2 quark helicity distribution $\Delta q(x)[7]$. The relations (3) and (4) indicate that one cannot find the partonic hard cross sections for the $F$-type and $D$-type functions independently. We use the "less singular" F-type functions as a complete set for the twist-3 distributions.

To present our formalism we focus on the single-spin-dependent cross section in SIDIS, $e(\ell)+p^{\uparrow}\left(p, S_{\perp}\right) \rightarrow e\left(\ell^{\prime}\right)+\pi\left(P_{h}\right)+X$, and consider the contribution associated with the twist-3 distribution in the transversely polarized nucleon. Here the twist-2 fragmentation function for the final pion is immediately factorized from the hadronic tensor $W_{\mu \nu}\left(p, q, P_{h}\right)$ ( $q=\ell-\ell^{\prime}$ is the momentum of the virtual photon) as

$$
W_{\mu \nu}\left(p, q, P_{h}\right)=\sum_{j=q, g} \int \frac{d z}{z^{2}} D_{j}(z) w_{\mu \nu}^{j}\left(p, q, \frac{P_{h}}{z}\right),
$$

where $D_{j}(z)$ is the quark and gluon fragmentation functions for the pion. To extract the twist-3 effect in $w_{\mu \nu}^{j}$, one has to analyze the diagrams shown in Fig. 1, where the momenta for the parton lines are assigned. The lower blobs in the figure represent the nucleon matrix elements. They are the Fourier transforms of the correlation functions which are schmatically written as $M^{(0)}(k) \sim\langle\bar{\psi} \psi\rangle$ and $M^{(1) \alpha}\left(k_{1}, k_{2}\right) \sim\left\langle\bar{\psi} A^{\alpha} \psi\right\rangle$, where $\langle\cdots\rangle \equiv\left\langle p S_{\perp}|\cdots| p S_{\perp}\right\rangle$. The middle blobs, $S^{(0)}\left(k, q, P_{h} / z\right)$ and $S_{\alpha}^{(1)}\left(k_{1}, k_{2}, q, P_{h} / z\right)$, are the partonic hard scattering parts, and the upper blobs represent the fragmentation function for the pion. To analyze these diagrams, we work in the Feynman gauge. A standard procedure to extract the twist3 effect is the collinear expansion in terms of the parton momenta $k$ and $k_{1,2}$ around the component pararell to the parent nucleon's momentum $p$. Since $M^{(1) \alpha=\perp}$ is suppressed by $1 / p^{+}$compared with $M^{(1) \alpha=+}$, one needs the collinear expansion only for the component $S_{+}^{(1)}=S_{\rho}^{(1)} p^{\rho} / p^{+}$for the right diagram of Fig. 1 .

In the approach by Qiu and Sterman [4] using Feynman gauge, $\partial^{\perp} A^{+}$appearing in the collinear expansion is identified as a part of the gluon's field strength $F^{\perp+}=\partial^{\perp} A^{+}-\partial^{+} A^{\perp}-$ 
$i g\left[A^{\perp}, A^{+}\right]$, and thus $\partial S_{\rho}^{(1)}\left(k_{1}, k_{2}, q, P_{h} / z\right) p^{\rho} /\left.\partial k_{2 \perp}^{\alpha}\right|_{k_{i}=x_{i} p}$, appearing as the coefficient of $\left\langle\bar{\psi}\left(\partial^{\perp} A^{+}\right) \psi\right\rangle$, was identified as the hard part for the $F$-type functions. In order to justify this procedure, it is necessary to show that " $-\partial^{+} A^{\perp}$ " also appears with exactly the same coefficient as that for $\partial^{\perp} A^{+}$. Note that, in the Feynman gauge, the matrix element of the type $\left\langle\bar{\psi} \partial^{\perp} A^{+} \psi\right\rangle$ is equally important as $\left\langle\bar{\psi} \partial^{+} A^{\perp} \psi\right\rangle$, and both matrix elements have to be treated as the same order in the collinear expansion. This is because, even though $\left\langle\bar{\psi} A^{+} \psi\right\rangle \gg\left\langle\bar{\psi} A^{\perp} \psi\right\rangle$ in the Feynman gauge in a frame with $p^{+} \gg p^{-}, p^{\perp}$, action of $\partial^{\perp}$ to the gluon field in $\left\langle\bar{\psi} A^{+} \psi\right\rangle$ brings relative suppression compared with $\partial^{+}$.

On the other hand, $A^{\perp}$ was identified as a part of $D^{\perp}=\partial^{\perp}-i g A^{\perp}$ in [4], and $S_{\perp}^{(1)}\left(x_{1} p, x_{2} p, q, P_{h} / z\right)$, appearing as the coefficient of $\left\langle\bar{\psi} A^{\perp} \psi\right\rangle$, was treated as the hard part for the $D$-type functions, independently from the $F$-type functions. However, if one identifies " $-\partial^{+} A^{\perp "}$ part in $F^{\perp+}$, it also affects the coefficients of $\left\langle\bar{\psi} A^{\perp} \psi\right\rangle$.

In this way, the twist-3 formalism presented by [4] was not complete, in particular, the gauge invariance and uniqueness of factorization formula was not shown explicitly.

Above consideration forces us to reorganize the collinear expansion. Since $F$-type and $D$-type functions are related as in (3) and (4), we take the approach of expressing the cross section in terms of $F$-type functions only. Detailed analysis of the diagrams shown in Fig. 1 shows that the single-spin-dependent cross section in the leading order perturbation theory arises solely from the right diagram in Fig.1. Taking into account the $-\partial^{+} A^{\perp}$ term in $F^{\perp+}$, we eventually arrive at the following result for $w_{\mu \nu}^{j}$ in (5):

$$
w_{\mu \nu}^{j} \quad=\int d x_{1} \int d x_{2} \operatorname{Tr}\left[\left.i \omega^{\alpha}{ }_{\beta} M_{F}^{\beta}\left(x_{1}, x_{2}\right) \frac{\partial S_{\rho}^{(1)}\left(k_{1}, k_{2}, q, P_{h} / z\right) p^{\rho}}{\partial k_{2}^{\alpha}}\right|_{k_{i}=x_{i} p}\right],
$$

with the consistency conditions

$$
\begin{aligned}
& \left.\left(x_{2}-x_{1}\right) \frac{\partial S_{\rho}^{(1)}\left(k_{1}, k_{2}, q, P_{h} / z\right) p^{\rho}}{\partial k_{2}^{\alpha}}\right|_{k_{i}=x_{i} p}+S_{\alpha}^{(1)}\left(x_{1} p, x_{2} p, q, P_{h} / z\right)=0, \\
& \left.\frac{\partial S_{\rho}^{(1)}\left(k_{1}, k_{2}, q, P_{h} / z\right) p^{\rho}}{\partial k_{1}^{\alpha}}\right|_{k_{i}=x_{i} p}+\left.\frac{\partial S_{\rho}^{(1)}\left(k_{1}, k_{2}, q, P_{h} / z\right) p^{\rho}}{\partial k_{2}^{\alpha}}\right|_{k_{i}=x_{i} p}=0
\end{aligned}
$$

for $\alpha=\perp$, where $M_{F}^{\beta}\left(x_{1}, x_{2}\right)$ is given in (1), $\omega^{\alpha \beta}=g^{\alpha \beta}-p^{\alpha} n^{\beta}$ and $\operatorname{Tr}[\cdots]$ indicates the Dirac trace, while color trace is implicit. In (6), the real quantity $w_{\mu \nu}^{j}$ is given as the pole contributions [3, 4] from internal propagators of the hard part $\partial S_{\rho}^{(1)}\left(k_{1}, k_{2}, q, P_{h} / z\right) p^{\rho} /\left.\partial k_{2}^{\alpha}\right|_{k_{i}=x_{i} p}$, which are classified as hard-pole (HP), soft-fermion-pole (SFP) and soft-gluon-pole (SGP) contributions. Since these three kinds of poles occur at different points in phase space, one can prove that each pole contribution satisfies the two conditions (7) and (8) separately, and the whole hadronic tensor $w_{\mu \nu}^{j}$ can be written in terms of the $F$-type functions only, including all the HP, SFP and SGP contributions. We emphasize the crucial role of the two conditions (7) and (8): Using these conditions for each pole contribution, the various terms associated with the gauge-noninvariant matrix elements, arising in the collinear expansion of $w_{\mu \nu}^{j}$, cancel out and only the term shown in (6) remains, which guarantees the factorization of the cross section and the gauge invariance of the formula. In particular, the first condition (7) can be proved using the Ward identities for color gauge invariance (see [2] for the detail). 
Pragmatically, the authors of [4] reached the same formula (6), but only for the SGP contribution. We emphasize, however, that our argument leading to (6) is totally different from [4], in particular, (6) is the principal formula for all HP, SFP and SGP contributions.

For the HP and SFP contributions in which $x_{1} \neq x_{2},(7)$ can be rewritten as

$$
\left.\frac{\partial S_{\rho}^{(1)}\left(k_{1}, k_{2}, q, P_{h} / z\right) p^{\rho}}{\partial k_{2}^{\alpha}}\right|_{k_{i}=x_{i} p}=\frac{1}{x_{1}-x_{2}} S_{\alpha}^{(1)}\left(x_{1} p, x_{2} p, q, P_{h} / z\right)
$$

Owing to this relation, one can obtain the relevant hard cross section in (6) also through $S_{\perp}^{(1)}\left(x_{1} p, x_{2} p, q, P_{h} / z\right)$, which is much simpler to calculate than the derivative of the amplitude, $\partial S_{\rho}^{(1)}\left(k_{1}, k_{2}, q, P_{h} / z\right) p^{\rho} /\left.\partial k_{2}^{\alpha}\right|_{k_{i}=x_{i} p}$. Since $S_{\perp}^{(1)}\left(x_{1} p, x_{2} p, q, P_{h} / z\right)$ does not contain any derivative, the HP and SFP contributions do not appear as the derivative terms, such as those $\propto d G_{F}\left(x_{b j}, x\right) / d x$ or $d \widetilde{G}_{F}\left(x_{b j}, x\right) / d x$ etc. For the SGP contribution, one has to calculate the derivative $\partial S_{\rho}^{(1)}\left(k_{1}, k_{2}, q, P_{h} / z\right) p^{\rho} /\left.\partial k_{2}^{\alpha}\right|_{k_{i}=x_{i} p}$, because of the lack of the relation (9) for $x_{1}=x_{2}$, and it gives rise to the derivative terms with $d G_{F}(x, x) / d x$. For the HP and SFP contributions, one can also rewrite the cross section in terms of $D$-type functions by using the relations (3) and (4) for $x_{1} \neq x_{2}$, without any subtlety.

We have applied the above formalism to SIDIS and derived [2] the complete singlespin-dependent cross section for $e p^{\uparrow} \rightarrow e \pi X$ associated with the twist-3 distributions for the transversely polarized nucleon, including all three kinds (HP, SFP and SGP) of pole contributions for $G_{F}\left(x_{1}, x_{2}\right)$ and $\widetilde{G}_{F}\left(x_{1}, x_{2}\right)$.

Our twist-3 formalism can be easily extended to other processes, such as Drell-Yan and direct photon productions, $p^{\uparrow} p \rightarrow \gamma^{(*)} X$, and the pion production in $p p$ collision, $p^{\uparrow} p \rightarrow \pi X$, which gives solid theoretical basis to the previously obtained cross section formula [4]-[10].

The work of K.T. was supported by the Grant-in-Aid for Scientific Research No. B19340063.

\section{References}

[1] Slides: http://indico.cern. ch/contributionDisplay. py? contribId=158\&sessionId=4\&conf Id=9499

[2] H. Eguchi, Y. Koike and K. Tanaka, Nucl. Phys. B763 198 (2007).

[3] A. V. Efremov and O. V. Teryaev, Sov. J. Nucl. Phys. 36140 (1982) [Yad. Phiz. 36242 (1982)]; Phys. Lett. B150 383 (1985).

[4] J. Qiu and G. Sterman, Nucl. Phys. B378 52 (1992).

[5] J. Qiu and G. Sterman, Phys. Rev. D59 014004 (1999).

[6] Y. Kanazawa and Y. Koike, Phys. Lett. B478 121 (2000); Phys. Lett. B490 (2000) 99; Phys. Rev. D64 034019 (2001).

[7] H. Eguchi, Y. Koike and K. Tanaka, Nucl. Phys. B752 1 (2006).

[8] X. D. Ji, J. W. Qiu, W. Vogelsang and F. Yuan, Phys. Rev. Lett. 97082002 (2006); Phys. Rev. D73 094017 (2006).

[9] X. D. Ji, J. W. Qiu, W. Vogelsang and F. Yuan, Phys. Lett. B638 178 (2006).

[10] C. Kouvaris, J. W. Qiu, W. Vogelsang, F. Yuan, Phys. Rev. D74 114013 (2006). 